$11-19-2002$

\title{
Synthesis of A Spacer-Armed Disulfated Tetrasaccharide of SB1a, A Carbohydrate Hapten Associated with Human Hepatocellular Carcinoma
}

Qin Li

Peking University

Hui Li

Peking University

Qing Li

Peking University

Qing Hua Lou

Peking University

Bin Su

Cleveland State University, B.SU@csuohio.edu

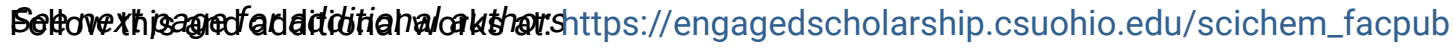

Part of the Chemistry Commons

How does access to this work benefit you? Let us know!

\section{Recommended Citation}

Li, Qin; Li, Hui; Li, Qing; Lou, Qing Hua; Su, Bin; Cai, Meng Shen; and Li, Zhong Jun, "Synthesis of A SpacerArmed Disulfated Tetrasaccharide of SB1a, A Carbohydrate Hapten Associated with Human Hepatocellular Carcinoma" (2002). Chemistry Faculty Publications. 420.

https://engagedscholarship.csuohio.edu/scichem_facpub/420

This Article is brought to you for free and open access by the Chemistry Department at EngagedScholarship@CSU. It has been accepted for inclusion in Chemistry Faculty Publications by an authorized administrator of EngagedScholarship@CSU. For more information, please contact library.es@csuohio.edu. 


\section{Authors}

Qin Li, Hui Li, Qing Li, Qing Hua Lou, Bin Su, Meng Shen Cai, and Zhong Jun Li 


\title{
Synthesis of a spacer-armed disulfated tetrasaccharide of $\mathrm{SB}_{1 \mathrm{a}}$, a carbohydrate hapten associated with human hepatocellular carcinoma
}

\author{
Qin Li, Hui Li, Qing Li, Qing-Hua Lou, Bin Su, Meng-Shen Cai, Zhong-Jun Li
}

\section{Introduction}

Aberrant cell-surface glycosylation is often closely associated with tumor progression and malignancy. ${ }^{1}$ In most cases, carbohydrate antigens may be rather specific to a certain type of tumor and are not overexpressed or recognized by the immune system in normal tissues. $^{2}$ Therefore, carbohydrate antigens have been greatly mesmerizing scientists in relevant fields because of their potential applications in tumor immunotherapy. ${ }^{3} \mathrm{SB}_{1 \mathrm{a}}$, a glycosphinolipid with a disulfated tetrasaccharide moiety, was first isolated from rat kidney by Tadano and Ishizuka. ${ }^{4}$ The normal human liver contains essentially no detectable amount of $\mathrm{SB}_{1 \mathrm{a}}$. However, studies have shown that a remarkable accumulation of $\mathrm{SB}_{1 \mathrm{a}}$ exists, not only in the cultured human hepatocellular carcinoma (HCC) cell lines, but also in glycolipid fractions extracted from HCC tissues. Therefore, it is suggested that $\mathrm{SB}_{1 \mathrm{a}}$ is one of the most important cancer-associated carbohydrate antigens of HCC. ${ }^{5,6}$

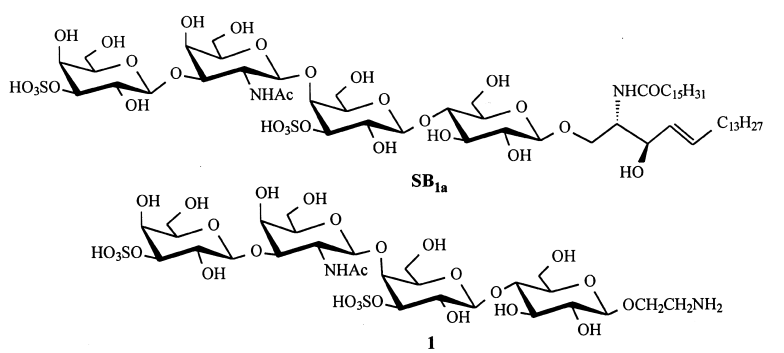

In order to elucidate the functions of $\mathrm{SB}_{1 \mathrm{a}}$ in detail, especially its mechanism involved in the onset, progression, and metastasis of $\mathrm{HCC}$, and hence pursue optimal carbohydrate-based anticancer vaccines for HCC, we have synthesized the disulfated tetrasaccharide moiety of the $\mathrm{SB}_{1 \mathrm{a}}$ determinant, namely compound $\mathbf{1}$, in which a 2-aminoethyl group is attached to the reducing terminal as a spacer arm, which could facilitate further formation of immunogenic glycoconjugates by the coupling of the spacer amino group and a carrier protein. 


\section{Results and discussion}

Of the various approaches available for the preparation of oligosaccharides, we adopted the stepwise synthetic strategy to build the target molecule. The reducing terminal D-lactosyl building block 3 of the target molecule was first synthesized in a good yield $(89.6 \%)$ via the regioselective etherification of the $3^{\prime}-\mathrm{OH}$ of 2-azidoethyl 2,3,6-tri- $O$-benzyl-2,6-di- $O$-benzyl- $\beta$-Dgalactopyranosyl-( $1 \rightarrow 4)$ - $\beta$-D-glucopyranoside

which was prepared steadily through several steps from

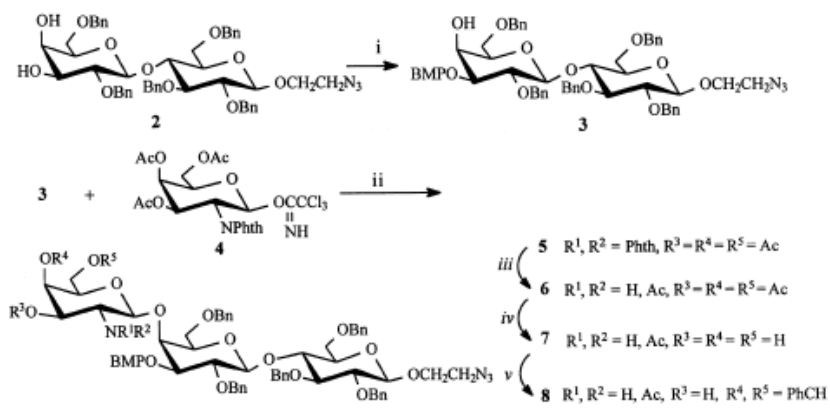

Scheme 1. Synthesis of the trisaccharide acceptor 8. (i) (a) $\mathrm{Bu}_{2} \mathrm{SnO}$, MeOH, reflux; (b) toluene, $p-\mathrm{CH}_{3} \mathrm{OC}_{6} \mathrm{H}_{4} \mathrm{CH}_{2} \mathrm{Cl}$, $\mathrm{Bu}_{4} \mathrm{NBr}, 4 \AA$ MS $\left(89.6 \%\right.$ ); (ii) TMSOTf, toluene, $-40^{\circ} \mathrm{C}$ $(83.2 \%)$; (iii) (a) 1,2-diaminoethane, $n$-butanol, $75^{\circ} \mathrm{C}$; (b) $\mathrm{Ac}_{2} \mathrm{O}-\mathrm{Py}\left(91.2 \%\right.$ ); (iv) $\mathrm{NaOMe}, \mathrm{MeOH}$; (v) $\mathrm{PhCH}(\mathrm{OMe})_{2}$, camphorsulfonic acid, $\mathrm{CH}_{3} \mathrm{CN}(90 \%)$.

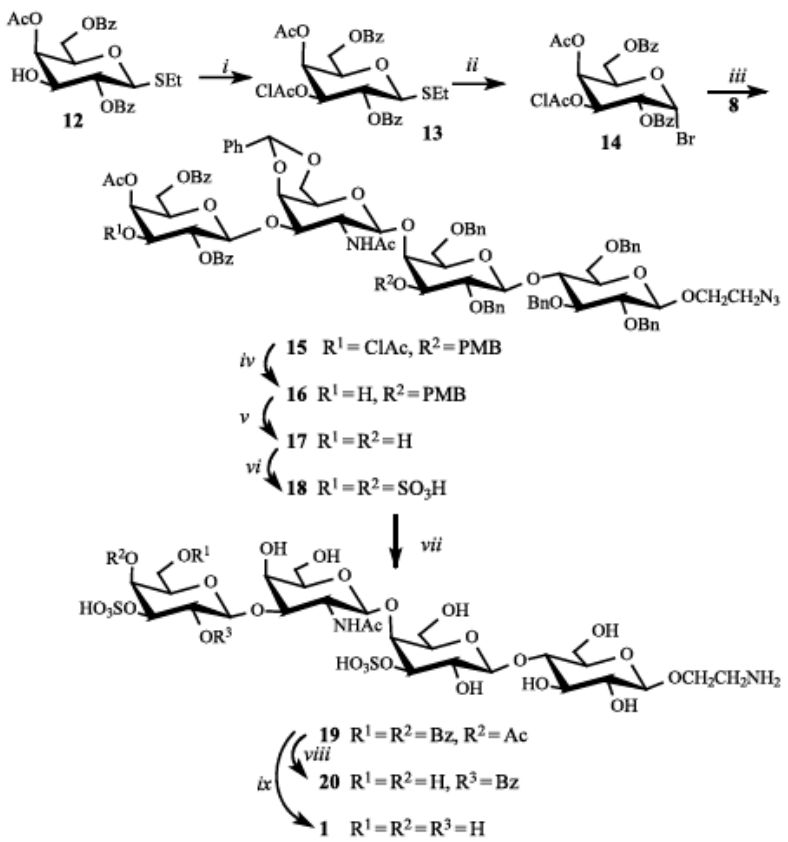

Scheme 2. Synthesis of the target tetrasaccharide. (i) $\mathrm{ClAcCl}$, $\mathrm{CH}_{2} \mathrm{Cl}_{2}$-pyridine $\left(83.7 \%\right.$ ); (ii) $\mathrm{Br}_{2}, \mathrm{CH}_{2} \mathrm{Cl}_{2}, 0^{\circ} \mathrm{C} \rightarrow \mathrm{rt}$; (iii) $\mathrm{AgOTf}, 4 \AA \mathrm{MS}, \mathrm{CH}_{2} \mathrm{Cl}_{2},-20^{\circ} \mathrm{C}(89 \%)$; (vi) thiourea, 2,6-lutidine, $\mathrm{CH}_{2} \mathrm{Cl}_{2}-\mathrm{EtOH}, 58^{\circ} \mathrm{C} \quad(76.4 \%)$; (v) CAN, $\mathrm{CH}_{3} \mathrm{CN}-\mathrm{H}_{2} \mathrm{O}$, rt, $\left(92.4 \%\right.$ ); (vi) $\mathrm{SO}_{3} \cdot \mathrm{Pyr}, \mathrm{Pyr}(95 \%)$; (vii) $\mathrm{H}_{2}$, $10 \% \mathrm{Pd} / \mathrm{C}, \mathrm{MeOH}-\mathrm{H}_{2} \mathrm{O}, 1 \mathrm{M} \mathrm{HCl}$; (viii) $0.012 \mathrm{M} \mathrm{NaOMe}-$ $\mathrm{MeOH}$, rt; (ix) $0.5 \mathrm{M} \mathrm{NaOMe}-\mathrm{MeOH}, 0{ }^{\circ} \mathrm{C}$.
D-lactose. ${ }^{7}$ In the synthesis of 3, the $p$-methoxybenzyl group (PMB) was introduced to the $3-\mathrm{OH}$ position of the galactosyl moiety via a dibutyltin oxide-mediated procedure ${ }^{8}$ followed by addition of $p$-methoxybenzyl chloride and tetrabutylammonium bromide in boiling toluene (Scheme 1). ${ }^{9}$

Standard glycosylation of $\mathbf{3}$ and the glycosyl donor 3,4,6-tri- $O$-acetyl-2-deoxy-2-phthalimido- $\beta$-D-galactopyranosyl trichloroacetimidate $(4)^{10}$ in toluene at $-40^{\circ} \mathrm{C}$ gave the desired $\beta$-linked trisaccharide 5 (83.2\%). Dephthaloylation of compound 5 with 1,2diaminoethane $\mathrm{e}^{11}$ in $n$-butanol at $75^{\circ} \mathrm{C}$, followed by acetylation, resulted in the formation of 6 . Subsequent O-deacetylation and benzylidenation at the C-4" and C-6" hydroxy groups with benzaldehyde dimethyl acetal in acetonitrile under acidic conditions provided the trisaccharide acceptor $\mathbf{8}$ in excellent yield.

However, for the assembly of the tetrasaccharide backbone, some interesting results occurred. In our initial design, ethyl 2,4,6-tri- $O$-acetyl-3- $O$ - $p$-methoxybenzyl-1-thio- $\beta$-D-galactopyranoside (9) or the corresponding glycosyl bromide $\mathbf{1 0}$ was chosen as the glycosyl donor to couple with the trisaccharide acceptor 8. No reaction occurred when $\mathbf{9}$ and $\mathbf{8}$ were mixed and stirred at room temperature in nitromethane or DMF employing $\mathrm{Bu}_{4} \mathrm{NBr}-\mathrm{CuBr}_{2}$ as the promoter. Neither did the coupling reaction of $\mathbf{9}$ and $\mathbf{8}$ using methyl triflate as the promoter in dichloromethane or diethyl ether. We next investigated the glycosylation of the donor $\mathbf{1 0}$ with the trisaccharide acceptor $\mathbf{8}$, no desired tetrasaccharide was obtained when silver triflate was chosen to promote the coupling reaction. The main product is the asymmetric $(1 \rightarrow 1)$-linked disaccharide 11, in which two galactosyl groups were condensed to each other by $\alpha$ and $\beta$ configurations at the anomeric center, respectively.

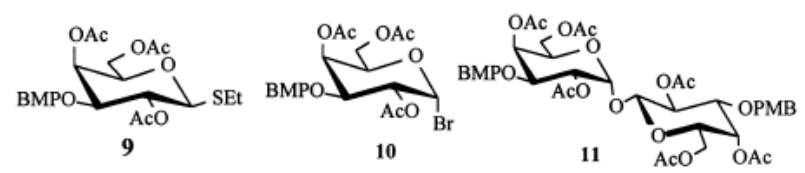

After a series of failures in the building of the tetrasaccharide backbone, we selected another type of glycosyl donor containing a benzoyl group at C-2 for coupling with acceptor $\mathbf{8}$. Therefore, we chose the glycosyl bromide 14 as the glycosyl donor. Compound $\mathbf{1 4}$ was synthesized by the in situ transformation of ethyl 4- $O$-acetyl-2,6-di- $O$-benzoyl-3- $O$-chloroacetyl-1-thio- $\beta$ D-galactopyranoside (13), which was prepared from chloroacetylation of the known ethyl 4- $O$-acetyl-2,6-di$O$-benzoyl-1-thio- $\beta$-D-galactopyranoside (12). ${ }^{12}$ To our surprise, the silver triflate-promoted glycosylation with 8 using donor 14 in dichloromethane at $-20^{\circ} \mathrm{C}$ gave the desired tetrasaccharide 15 in very high yield $(89 \%)$ (Scheme 2). 
Deblocking of $\mathbf{1 5}$ to the target tetrasaccharide $\mathbf{1}$ includes several steps as in the following. At first, selective removal of the chloroacetyl group at the $3^{\prime \prime \prime}$ $\mathrm{OH}$ position and the $p$-methoxybenzyl group at $3^{\prime}-\mathrm{OH}$ position with thiourea and cerium(IV) ammonium nitrate $(\mathrm{CAN})$, respectively, gave $\mathbf{1 7}$. Then, treatment of the diol 17 with sulfur trioxide-pyridine complex in pyridine furnished the disulfated compound $\mathbf{1 8}$ in $95 \%$ yield. But deprotection of $\mathbf{1 8}$ was rather complicated. Catalytic hydrogenolysis, using palladium-on-charcoal in different solvents $(\mathrm{AcOH}, 2: 1 \mathrm{MeOH}-\mathrm{AcOH})$ was sluggish and the yield was low. This problem may be ascribed to the catalyst passiveness due to the interaction with the aminoethyl fragment formed. A similar phenomenon has been observed by Spijker et al. ${ }^{13}$ and Stahl et al. ${ }^{14}$ To avoid this inhibitory effect, hydrochloric acid was added to the reaction mixture to convert the formed amine to its hydrochloride salt. This greatly increased the hydrogenolysis rate and yield. Then, deacylation of 19 with $0.012 \mathrm{M}$ sodium methoxide in $\mathrm{MeOH}$ at room temperature provided product $\mathbf{2 0}$ with the $2^{\prime \prime \prime}-O$-benzoyl group retained. Increasing base concentration and prolonging reaction time only led to decomposition of the product. When the O-deacylation was carried out with ammonia in $\mathrm{MeOH}$, no O-deacylation but O-desulfonation was observed. Finally, the saponification of 19 was completed with $0.5 \mathrm{M}$ sodium methoxide in $\mathrm{MeOH}$ at $0{ }^{\circ} \mathrm{C}$ for $6 \mathrm{~h}$ to give the title compound $\mathbf{1}$ in $90 \%$ yield.

\section{Experimental}

General methods.-All moisture-sensitive reactions were performed under argon atmosphere, and organic solvents were dried over standard drying agents and freshly distilled prior to use. Optical rotations were measured at $25^{\circ} \mathrm{C}$ with an Optical Activity LTD AA$10 \mathrm{R}$ polarimeter in a $5-\mathrm{cm}, 1-\mathrm{mL}$ cell. Melting points were uncorrected. NMR spectra were recorded at room temperature with a JEOL 300, Bruker AM 400, and INOVA-600 spectrometers. Chemical shifts were expressed in ppm downfield from the signal for internal $\mathrm{Me}_{4} \mathrm{Si}$ for solutions in $\mathrm{CDCl}_{3}, \mathrm{CD}_{3} \mathrm{OD}$ and DMSO- $d_{6}$, or DSS in case of $\mathrm{D}_{2} \mathrm{O}$. MALDI-TOFMS analyses were performed with an LDI-1700 mass spectrometer. Column chromatography was performed on silica gel $\mathrm{H}$ 60 , and fractions were monitored by TLC on silica gel $60 \mathrm{GF}_{254}$ with detection by UV light and/or by charring with $10 \% \mathrm{H}_{2} \mathrm{SO}_{4}$ in EtOH. Solutions were concentrated at or below $40{ }^{\circ} \mathrm{C}$ and dried with anhydrous $\mathrm{Na}_{2} \mathrm{SO}_{4}$.

2-Azidoethyl 2,6-di-O-benzyl-3-O-p-methoxybenzyl- $\beta$ - D-galactopyranosyl- $(1 \rightarrow 4)$ - 2,3,6-tri-O - benzyl- $\beta$ - Dglucopyranoside (3). - To a solution of 2-azidoethyl 2,6di- $O$-benzyl- $\beta$-D-galactopyranosyl- $(1 \rightarrow 4)$-2,3,6-tri- $O$ benzyl- $\beta$-D-glucopyranoside $\left(2,{ }^{7} 3.53 \mathrm{~g}, 4.10 \mathrm{mmol}\right)$ in dry $\mathrm{MeOH}(200 \mathrm{~mL})$ was added $\mathrm{Bu}_{2} \mathrm{SnO}(1.77 \mathrm{~g}, 7.11$ $\mathrm{mmol}$ ), and the mixture was stirred overnight at $60^{\circ} \mathrm{C}$ under Ar. After cooling to room temperature, the mixture was concentrated, and the residue was dissolved in dry toluene $(200 \mathrm{~mL}) . \mathrm{Bu}_{4} \mathrm{NBr}(0.588 \mathrm{~g}, 1.8 \mathrm{mmol})$ and powdered $4 \AA$ molecular sieves (5 g) were added, and the mixture was stirred for $1 \mathrm{~h}$ at room temperature under Ar. Then, $p$-methoxybenzyl chloride $(1.77 \mathrm{~mL})$ was added, and the mixture was stirred for $4 \mathrm{~h}$ at $120^{\circ} \mathrm{C}$, at the end of which time TLC (5:1 tolueneEtOAc) showed the disappearance of $\mathbf{2}$ and the formation of 3. After cooling to room temperature, $\mathrm{MeOH}$ $(10 \mathrm{~mL})$ and $\mathrm{Et}_{3} \mathrm{~N}(2.5 \mathrm{~mL})$ were added, and the stirring was continued for $15 \mathrm{~min}$. After filtration through Celite, the filtrate was concentrated. Column chromatography (4:1 petroleum ether-acetone) of the residue afforded 3 as a white needles $(3.60 \mathrm{~g}, 89.6 \%)$. $\mathrm{mp} 86.0-87.0^{\circ} \mathrm{C} .[\alpha]_{\mathrm{D}}+15.8^{\circ}\left(\mathrm{c} 1.58, \mathrm{CHCl}_{3}\right) .{ }^{1} \mathrm{H}$ NMR $\left(\mathrm{CDCl}_{3}\right): \delta 7.51-6.91(\mathrm{~m}, 29 \mathrm{H}, \mathrm{Ar}-\mathrm{H}), 5.12-$ $3.43\left(\mathrm{~m}, 32 \mathrm{H}\right.$, sugar $\mathrm{H}, 5 \times \mathrm{PhCH}_{2}, \mathrm{CH}_{3} \mathrm{OC}_{6} \mathrm{H}_{4} \mathrm{CH}_{2}$, $\mathrm{OCH}_{2} \mathrm{CH}_{2} \mathrm{~N}_{3}$ ), 2.64 (bs, $1 \mathrm{H}, 4^{\prime}-\mathrm{OH}$ ). ${ }^{13} \mathrm{C}$ NMR: $\delta$ $159.1,113.6\left(\mathrm{CH}_{3} \mathrm{OC}_{6} \mathrm{H}_{4} \mathrm{CH}_{2}\right), 138.9,138.5,138.4$, $138.0,129.8,129.2,128.2,127.9,127.8,127.5,127.4$, 127.3, 127.1 (Ar-C), 103.4, 102.4 (C-1, C-1'), 82.6, 81.6, 79.2, 76.3, 75.1, 75.0, 74.9, 73.3, 72.9, 72.7, 71.7, 68.3, 68.0, 67.9, 65.9 (sugar $\mathrm{C}, 5 \times \mathrm{PhCH}_{2}, \mathrm{CH}_{3} \mathrm{OC}_{6} \mathrm{H}_{4} \mathrm{CH}_{2}$, $\left.\mathrm{OCH}_{2} \mathrm{CH}_{2} \mathrm{~N}_{3}\right), 55.0\left(\mathrm{OCH}_{3}\right), 50.7\left(\mathrm{CH}_{2} \mathrm{~N}_{3}\right)$. MALDITOFMS: $m / z \quad 1002.8[\mathrm{M}+\mathrm{Na}]^{+}$. Anal. Calcd for $\mathrm{C}_{17} \mathrm{H}_{29} \mathrm{~N}_{3} \mathrm{O}_{17}: \mathrm{C}, 69.72 ; \mathrm{H}, 6.42 ; \mathrm{N}, 3.79$. Found: $\mathrm{C}$, $69.40 ; \mathrm{H}, 6.56 ; \mathrm{N}, 3.79$.

2-Azidoethyl 3,4,6-tri-O-acetyl-2-deoxy-2-phthalimido- $\beta$-D-galactopyranosyl- $(1 \rightarrow 4)$ - 2,6-di-O-benzyl-3$\mathrm{O}$-p-methoxybenzyl- $\beta$-D-galactopyranosyl- $(1 \rightarrow 4)-2,3,6$ tri-O-benzyl- $\beta$-D-glucopyranoside (5). - To a solution of $3(1.00 \mathrm{~g})$ and 3,4,6-tri- $O$-acetyl-2-deoxy-2-phthalimido- $\beta$-D-galactopyranosyl trichloroacetimidate $\mathbf{( 4 ,}{ }^{10}$ $1.00 \mathrm{~g})$ in dry toluene $(50 \mathrm{~mL})$ were added $4 \AA$ molecular sieves $(0.93 \mathrm{~g})$, and the mixture was stirred for $1 \mathrm{~h}$ under argon. The mixture was cooled to $-40{ }^{\circ} \mathrm{C}$, and a solution of TMSOTf $(60 \mu \mathrm{L})$ in dry $\mathrm{CH}_{2} \mathrm{Cl}_{2}(1 \mathrm{~mL})$ was added. The mixture was stirred at $-40^{\circ} \mathrm{C}$ for $3 \mathrm{~h}$ and then overnight at room temperature. $\mathrm{Et}_{3} \mathrm{~N}(0.5$ $\mathrm{mL}$ ) was added, and the mixture was diluted with EtOAc $(100 \mathrm{~mL})$ and filtered (Celite). The filtrate was washed with water $(100 \mathrm{~mL})$, aq $\mathrm{NaHCO}_{3}(100 \mathrm{~mL})$ and water $(100 \mathrm{~mL})$, dried, concentrated. Column chromatography (4:1:0.1 $\mathrm{C}_{6} \mathrm{H}_{12}-\mathrm{CHCl}_{3}$-acetone) afforded 5 , isolated as a colorless foam $(1.12 \mathrm{~g}, 83.2 \%)$. $[\alpha]_{\mathrm{D}}$ $+8.5^{\circ}\left(c\right.$ 1.42, $\left.\mathrm{CHCl}_{3}\right) .{ }^{1} \mathrm{H}$ NMR $\left(\mathrm{CDCl}_{3}\right): \delta 7.87-6.79$ (m, $33 \mathrm{H}, 5 \times \mathrm{PhCH}_{2}$, Phth, $\mathrm{CH}_{3} \mathrm{OC}_{6} \mathrm{H}_{4} \mathrm{CH}_{2} \mathrm{O}$ ), 6.61 (dd, $\left.1 \mathrm{H}, J_{3^{\prime \prime \prime}, 4^{\prime \prime}} 3.90 \mathrm{~Hz}, J_{2^{\prime}, 3^{\prime \prime}} 11.70 \mathrm{~Hz}, \mathrm{H}-3^{\prime \prime}\right), 5.55$ (d, $\left.1 \mathrm{H}, \mathrm{H}-4^{\prime \prime}\right), 5.35$ (d, $\left.1 \mathrm{H}, J_{1^{\prime \prime}, 2^{\prime \prime}} 8.40 \mathrm{~Hz}, \mathrm{H}-1^{\prime \prime}\right), 3.79$ (s, $\left.3 \mathrm{H}, \mathrm{CH}_{3} \mathrm{O}\right), 2.20,2.03,1.85(3 \mathrm{~s}, 3 \mathrm{H}$ each, $3 \times \mathrm{OAc})$. ${ }^{13} \mathrm{C}$ NMR $\left(\mathrm{CDCl}_{3}\right): \delta 170.4,170.3,169.8,168.2,167.4$ (Phth, $3 \times \mathrm{OAc}), \quad 159.4,113.7 \quad\left(\mathrm{CH}_{3} \mathrm{OC}_{6} \mathrm{H}_{4} \mathrm{CH}_{2} \mathrm{O}\right)$, $139.1,138.9,138.7,138.4,138.3,134.0,133.7,132.6$, 
$131.7,130.1,130.0,129.7,28.3,128.2,128.0,127.9$, 127.5, 127.4, 127.3, 123.4, 123.3 (Ar-C), 103.7, 102.1

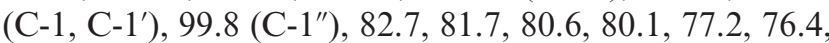
76.1, 75.7, 75.3, 75.1, 74.6, 73.2, 73.1, 72.4, 70.4, 68.9, 68.2, 68.1, 67.4, 66.6, 61.2 (sugar $\mathrm{C}, \mathrm{CH}_{3} \mathrm{OC}_{6} \mathrm{H}_{4} \mathrm{CH}_{2}$, $\left.5 \times \mathrm{PhCH}_{2}, \mathrm{OCH}_{2} \mathrm{CH}_{2} \mathrm{~N}_{3}\right), 55.3\left(\mathrm{CH}_{3} \mathrm{O}\right), 51.5\left(\mathrm{C}-2^{\prime \prime}\right)$, $50.9\left(\mathrm{CH}_{2} \mathrm{~N}_{3}\right), 20.7,20.5,20.4(3 \times \mathrm{OAc})$. Anal. Calcd for $\mathrm{C}_{77} \mathrm{H}_{82} \mathrm{~N}_{4} \mathrm{O}_{21}: \mathrm{C}, 66.04 ; \mathrm{H}, 5.87 ; \mathrm{N}, 4.01$. Found: $\mathrm{C}$, $65.90 ; \mathrm{H}, 6.13 ; \mathrm{N}, 3.74$.

2-Azidoethyl 2-acetamido-3,4,6-tri-O-acetyl-2-deoxy$\beta$-D-galactopyranosyl- $(1 \rightarrow 4)-2,6-d i-\mathrm{O}-$ benzyl-3-O-pmethoxybenzyl- $\beta$-D-galactopyranosyl- $(1 \rightarrow 4)$ - 2,3,6-triO-benzyl- $\beta$-D-glucopyranoside (6). - A solution of $\mathbf{5}$ $(2.30 \mathrm{~g}, 1.65 \mathrm{mmol})$ and 1,2-diaminoethane $(32 \mathrm{~mL})$ in $n$-butanol $(117 \mathrm{~mL})$ was stirred overnight at $75^{\circ} \mathrm{C}$ under argon. After cooling to room temperature, the mixture was co-evaporated with toluene $(3 \times 50 \mathrm{~mL})$. A solution of the residue in 1:1 $\mathrm{Ac}_{2} \mathrm{O}$-pyridine $(130 \mathrm{~mL})$ was stirred overnight at room temperature, then the mixture was co-evaporated with toluene $(3 \times 30 \mathrm{~mL})$. Column chromatography of the residue (2.3:1 petroleum ether-acetone) afforded $\mathbf{6}$, isolated as a colorless solid (1.96 g, 91.2\%). $[\alpha]_{\mathrm{D}}+12.6^{\circ}$ (c 1.27, $\left.\mathrm{CHCl}_{3}\right) .{ }^{1} \mathrm{H}$ NMR $\left(\mathrm{CDCl}_{3}\right): \delta 7.47-6.90(\mathrm{~m}, 29 \mathrm{H}$, $\left.5 \times \mathrm{PhCH}_{2}, \mathrm{CH}_{3} \mathrm{OC}_{6} \mathrm{H}_{4} \mathrm{CH}_{2}\right), 3.84\left(\mathrm{~s}, 3 \mathrm{H}, \mathrm{CH}_{3} \mathrm{O}\right)$, 2.18, 2.00, 1.93 (3 s, $3 \mathrm{H}$ each, $3 \times \mathrm{OAc}), 1.69$ (s, $3 \mathrm{H}$, NHAc). ${ }^{13} \mathrm{C}$ NMR $\left(\mathrm{CDCl}_{3}\right): \delta 170.3,170.2,169.7(4 \times$ Ac), 159.8, $114.3\left(\mathrm{CH}_{3} \mathrm{OC}_{6} \mathrm{H}_{4} \mathrm{CH}_{2}\right), 103.5,103.1,102.5$ $\left(\mathrm{C}-1,1^{\prime}, 1^{\prime \prime}\right), 55.3\left(\mathrm{CH}_{3} \mathrm{O}\right), 50.8,50.7\left(\mathrm{C}-2^{\prime \prime}, \mathrm{CH}_{2} \mathrm{~N}_{3}\right)$, 23.2 (NHAc), 20.8, 20.6, $20.5(3 \times \mathrm{OAc})$. Anal. Calcd for $\mathrm{C}_{71} \mathrm{H}_{82} \mathrm{~N}_{4} \mathrm{O}_{20}: \mathrm{C}, 65.04 ; \mathrm{H}, 6.26 ; \mathrm{N}, 4.27$. Found: $\mathrm{C}$, 65.39; H, 6.38; N, 4.21.

2-Azidoethyl 2-acetamido-4,6-O-benzylidene-2-deoxy- $\beta$-D-galactopyranosyl- $(1 \rightarrow 4)-2,6$ - di-O-benzyl-3$\mathrm{O}$-p-methoxybenzyl- $\beta$-D-galactopyranosyl- $(1 \rightarrow 4)-2,3,6$ tri-O-benzyl- $\beta$-D-glucopyranoside (8). - To a solution of $6(1.30 \mathrm{~g}, 0.992 \mathrm{mmol})$ in dry $\mathrm{MeOH}(40 \mathrm{~mL})$ was added $\mathrm{NaOMe}(108 \mathrm{mg})$. The mixture was stirred at room temperature for $14 \mathrm{~h}$, then neutralized with cation-exchange resin $\left(\mathrm{H}^{+}\right.$form $)$, and filtered. The filtrate was concentrated affording 7 (1.25 g, quant). To a solution of $7(1.25 \mathrm{~g})$ and $\alpha, \alpha$-dimethoxytoluene (354 $\mu \mathrm{L})$ in dry $\mathrm{CH}_{3} \mathrm{CN}(15 \mathrm{~mL})$ was added camphorsulfonic acid until $\mathrm{pH} 4$ was attained. The mixture was stirred at room temperature for $24 \mathrm{~h}$, then $\mathrm{Et}_{3} \mathrm{~N}(0.5$ $\mathrm{mL}$ ) was added, and the solvent was removed under reduced pressure. The residue was chromatographed (2:1 petroleum ether-acetone) to give $8(1.21 \mathrm{~g}, 90 \%)$ : ${ }^{1} \mathrm{H}$ NMR $\left(\mathrm{CDCl}_{3}\right): \delta 7.59-6.79(\mathrm{~m}, 34 \mathrm{H}, \mathrm{Ar}-\mathrm{H}), 5.59$ (s, $1 \mathrm{H}, \mathrm{PhCH}), 4.93-3.35(\mathrm{~m}, 40 \mathrm{H}$, sugar $\mathrm{H}, 5 \times$ $\left.\mathrm{PhCH}_{2}, \mathrm{CH}_{3} \mathrm{OC}_{6} \mathrm{H}_{4} \mathrm{CH}_{2}, \mathrm{OCH}_{2} \mathrm{CH}_{2} \mathrm{~N}_{3}\right), 1.65$ (s, $3 \mathrm{H}$, NHAc). ${ }^{13} \mathrm{C} \mathrm{NMR}\left(\mathrm{CDCl}_{3}\right): \delta 173.6$ (NHAc), 159.9, $114.1\left(\mathrm{CH}_{3} \mathrm{OC}_{6} \mathrm{H}_{4} \mathrm{CH}_{2}\right), 138.5,138.4,138.1,137.9$, $137.8,130.2,129.6,128.8,128.7,128.6,128.5,128.4$, $128.2,128.0,127.9,127.7,127.5,127.2,126.4$ (Ar-C), $101.1(\mathrm{PhCH}), 103.5,102.9,102.5\left(\mathrm{C}-1, \mathrm{C}-1^{\prime}, \mathrm{C}-1^{\prime \prime}\right)$,
$82.4,81.9,81.5,80.6,76.5,75.9,75.5,75.1,74.7,74.4$, 73.1, 73.0, 72.7, 68.8, 68.0, 67.1, 61.1 (sugar $\mathrm{C}$, $\left.\mathrm{CH}_{3} \mathrm{OC}_{6} \mathrm{H}_{4} \mathrm{CH}_{2}, 5 \times \mathrm{PhCH}_{2}, \mathrm{OCH}_{2} \mathrm{CH}_{2} \mathrm{~N}_{3}\right), 55.2,55.1$ $\left(\mathrm{CH}_{3} \mathrm{O}, \mathrm{C}-2^{\prime \prime}\right), 50.8\left(\mathrm{CH}_{2} \mathrm{~N}_{3}\right), 22.4$ (NHAc). MALDITOFMS: $1294.0[\mathrm{M}+\mathrm{Na}]^{+}, 1310[\mathrm{M}+\mathrm{K}]^{+}$. Anal. Calcd for $\mathrm{C}_{72} \mathrm{H}_{80} \mathrm{~N}_{4} \mathrm{O}_{17}$ : C, 67.92; $\mathrm{H}, 6.29 ; \mathrm{N}, 4.40$. Found: C, 67.76; H, 6.50; N, 4.39.

Ethyl 4-O-acetyl-2,6-di-O-benzoyl-3-O-chloroacetyl1-thio- $\beta$-D-galactopyranoside (13). - Monochloroacetyl chloride $(0.53 \mathrm{~mL}, 6.67 \mathrm{mmol})$ in dry $\mathrm{CH}_{2} \mathrm{Cl}_{2}(10 \mathrm{~mL})$ was added dropwise to a cooled $\left(0{ }^{\circ} \mathrm{C}\right)$ solution of ethyl 4- $O$-acetyl-2,6-di- $O$-benzoyl-1-thio- $\beta$-D-galactopyranoside $\left(\mathbf{1 2},{ }^{12} 2.22 \mathrm{~g}, 4.26 \mathrm{mmol}\right)$ in $5: 1 \mathrm{CH}_{2} \mathrm{Cl}_{2}$-pyridine $(60 \mathrm{~mL})$. After $2 \mathrm{~h}$ the solution was washed with $\mathrm{H}_{2} \mathrm{O}$, dried, filtered and concentrated. After column chromatography (7:1 petroleum ether-EtOAc) $\mathbf{1 3}$ was obtained: $[\alpha]_{\mathrm{D}}+7.1^{\circ}\left(c 1.13, \mathrm{CHCl}_{3}\right) .{ }^{1} \mathrm{H}$ NMR $\left(\mathrm{CDCl}_{3}\right)$ : $\delta$ 7.99-7.40 (m, $10 \mathrm{H}, \mathrm{Ar}-\mathrm{H}), 5.60-5.52(\mathrm{~m}, 2 \mathrm{H}, \mathrm{H}-2$, 4), $5.36\left(\mathrm{dd}, 1 \mathrm{H}, J_{2,3} 9.90 \mathrm{~Hz}, J_{3,4} 3.30 \mathrm{~Hz}, \mathrm{H}-3\right), 4.72$ $\left(\mathrm{d}, 1 \mathrm{H}, J_{1,2} 9.90 \mathrm{~Hz}, \mathrm{H}-1\right), 4.54\left(\mathrm{dd}, 1 \mathrm{H}, J_{5,6 \mathrm{a}} 6.60 \mathrm{~Hz}\right.$, $\left.J_{6 \mathrm{a}, 6 \mathrm{~b}} 11.40 \mathrm{~Hz}, \mathrm{H}-6 \mathrm{a}\right), 4.32\left(\mathrm{dd}, 1 \mathrm{H}, J_{5,6 \mathrm{~b}} 6.90 \mathrm{~Hz}\right.$, $\mathrm{H}-6 \mathrm{~b}), 3.88$ (m, $\left.2 \mathrm{H}, \mathrm{ClCH}_{2} \mathrm{CO}\right), 2.77-2.68(\mathrm{~m}, 2 \mathrm{H}$, $\mathrm{CH}_{3} \mathrm{CH}_{2} \mathrm{~S}$ ), 2.19 (s, $3 \mathrm{H}, \mathrm{Ac}$ ), 1.23 (t, $3 \mathrm{H}, \mathrm{CH}_{3} \mathrm{CH}_{2} \mathrm{~S}$ ). ${ }^{13} \mathrm{C}$ NMR $\left(\mathrm{CDCl}_{3}\right): \delta 170.4,166.6,165.9,165.2(\mathrm{CO})$, 133.5 (2 C), 129.8, 129.6, 129.2, 129.0, 128.5 (Ar-C), 84.2 (C-1), 74.4, 73.6, 67.7, 67.3, 61.7 (C-2, 3, 4, 5, 6), $40.3\left(\mathrm{ClCH}_{2} \mathrm{CO}\right), 24.6\left(\mathrm{CH}_{3} \mathrm{CH}_{2} \mathrm{~S}\right), 20.7$ (Ac), 14.8 $\left(\mathrm{CH}_{3} \mathrm{CH}_{2} \mathrm{~S}\right)$.

2-Azidoethyl 4-O-acetyl-2,6-di-O-benzoyl-3-Ochloroacetyl - $\beta$ - D - galactopyranosyl- $(1 \rightarrow 3)$ - 2-acetamido-4,6-O-benzylidene-2-deoxy- $\beta$-D-galactopyranosyl-(1 $\rightarrow 4$ )-2,6-di-O-benzyl-3-O-p-methoxybenzyl- $\beta$-D-galactopyranosyl-( $(\rightarrow 4)$-2,3,6-tri-O-benzyl- $\beta$-D-glucopyranoside (15). - To a solution of $13(402 \mathrm{mg}, 0.731 \mathrm{mmol})$ in dry $\mathrm{CH}_{2} \mathrm{Cl}_{2}(16 \mathrm{~mL})$ was added $\mathrm{Br}_{2}(35 \mu \mathrm{L}, 0.731$ $\mathrm{mmol})$ at $0{ }^{\circ} \mathrm{C}$. The solution was stirred at $0{ }^{\circ} \mathrm{C}$ for 40 min, and the solvent was subsequently evaporated. After co-evaporation twice with benzene, the residue was dissolved in $\mathrm{CH}_{2} \mathrm{Cl}_{2}(16 \mathrm{~mL})$ and added to a mixture of 8 (560 mg, $0.440 \mathrm{mmol})$, AgOTf $(268 \mathrm{mg})$, 2,6-di-tert-butyl-4-methylpyridine $(94.6 \mathrm{mg})$ and crushed $4 \AA$ molecular sieves $(950 \mathrm{mg})$ in $\mathrm{CH}_{2} \mathrm{Cl}_{2}(16$ $\mathrm{mL}$ ), which had been stirred under argon for $40 \mathrm{~min}$, and then cooled to $-20^{\circ} \mathrm{C}$. The mixture was allowed to warm to room temperature and to stir overnight. The reaction mixture was diluted with $\mathrm{CH}_{2} \mathrm{Cl}_{2}(100$ $\mathrm{mL}$ ) and filtered through Celite. The filtrate was washed with aq $\mathrm{NaHCO}_{3}, 10 \% \mathrm{Na}_{2} \mathrm{~S}_{2} \mathrm{O}_{3}$ and $\mathrm{H}_{2} \mathrm{O}$. The organic layer was dried and concentrated. Column chromatography (2.3:1 petroleum ether-acetone) of the residue afforded $15(690 \mathrm{mg}, 89.0 \%)$ as a white solid. $[\alpha]_{\mathrm{D}}+41.2^{\circ}\left(c \quad 0.97, \mathrm{CHCl}_{3}\right) \cdot{ }^{1} \mathrm{H}$ NMR $\left(\mathrm{CDCl}_{3}\right): \delta$ 8.08-6.73 (m, $44 \mathrm{H}, \mathrm{Ar}-\mathrm{H}), 5.56-3.23(\mathrm{~m}, 50 \mathrm{H}, \mathrm{PhCH}$, $5 \times \mathrm{PhCH}_{2}, \mathrm{CH}_{3} \mathrm{OC}_{6} \mathrm{H}_{4} \mathrm{CH}_{2}, \mathrm{ClCH}_{2} \mathrm{CO}, \mathrm{OCH}_{2} \mathrm{CH}_{2} \mathrm{~N}_{3}$ and sugar $\mathrm{H}$ ), 2.24 (s, $3 \mathrm{H}, \mathrm{OAc}), 1.30$ (s, $3 \mathrm{H}, \mathrm{NHAc}$ ). ${ }^{13} \mathrm{C} \mathrm{NMR}\left(\mathrm{CDCl}_{3}\right): \delta 171.4,170.9,167.0,166.3,165.1$ 
$\left(2 \times \mathrm{PhCO}, \mathrm{ClCH}_{2} \mathrm{CO}, \quad \mathrm{NHAc}, \quad \mathrm{OAc}\right), \quad 159.6,114.1$ $\left(\mathrm{CH}_{3} \mathrm{OC}_{6} \mathrm{H}_{4} \mathrm{CH}_{2}\right), 138.9,138.7,138.6,138.5,133.9$, $130.7,130.2,130.0,129.8,129.7,129.0,128.9,128.8$, $128.7,128.6,128.4,128.0,127.9,127.6126 .7$ (Ar-C), 104.0, 102.8, 102.6, 101.1, 99.9 (C-1, C-1', C-1", C-1"', $\mathrm{PhCH}), 83.2,82.0,80.4,76.6,75.6,75.4,73.0,72.5$, 71.1, 69.6 (sugar $\mathrm{C}, \quad 5 \times \mathrm{PhCH}_{2}, \mathrm{CH}_{3} \mathrm{OC}_{6} \mathrm{H}_{4} \mathrm{CH}_{2}$,

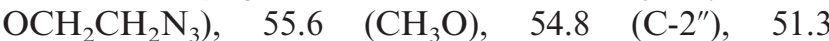
$\left(\mathrm{OCH}_{2} \mathrm{CH}_{2} \mathrm{~N}_{3}\right), 40.7\left(\mathrm{ClCH}_{2} \mathrm{CO}\right), 23.7$ (NHAc), 21.2 (OAc). MALDI-TOFMS: $m / z 1783.5[\mathrm{M}+\mathrm{Na}]^{+}$. Anal. Calcd for $\mathrm{C}_{96} \mathrm{H}_{102} \mathrm{ClN}_{4} \mathrm{O}_{26}$ : C, 65.45; H, 5.80; N, 3.18. Found: C, 65.41; H, 6.01; N, 3.15.

2-Azidoethyl 4-O-acetyl-2,6-di-O-benzoyl-3-Ochloroacetyl - $\beta$ - D - galactopyranosyl- $(1 \rightarrow 3)$ - 2 - acetamido-4,6-O-benzylidene-2-deoxy- $\beta$-D-galactopyranosyl-(1 $\rightarrow 4)$ - 2,6-di-O-benzyl- $\beta$ - D-galactopyranosyl - $(1 \rightarrow 4)$ 2,3,6-tri-O-benzyl- $\beta$-D-glucopyranoside (16). - To a solution of $15(575 \mathrm{mg}, 0.327 \mathrm{mmol})$ in $1: 1 \mathrm{CH}_{2} \mathrm{Cl}_{2}-\mathrm{abs}$ EtOH $(16 \mathrm{~mL})$ was added thiourea $(115 \mathrm{mg})$ and 2,6-lutidine $(69 \mu \mathrm{L})$. After stirring for $10 \mathrm{~h}$ at $58^{\circ} \mathrm{C}$, the mixture was cooled to room temperature and diluted with $\mathrm{CH}_{2} \mathrm{Cl}_{2}$ and washed with water. The organic layer was dried and concentrated. Purification of the residue by chromatography $(2: 1: 0.1$ petroleum ether-EtOAc$\mathrm{EtOH})$ gave $16(420 \mathrm{mg}, 76.4 \%)$. $[\alpha]_{\mathrm{D}}+21.1^{\circ}(c 1.14$, $\left.\mathrm{CHCl}_{3}\right) .{ }^{1} \mathrm{H}$ NMR $\left(\mathrm{CDCl}_{3}\right): \delta 8.06-6.80(\mathrm{~m}, 44 \mathrm{H}$, Ar-H), 5.45-3.12 (m, $48 \mathrm{H}, \quad \mathrm{PhCH}_{2}, \mathrm{PhCH}$, $\mathrm{CH}_{3} \mathrm{OC}_{6} \mathrm{H}_{4} \mathrm{CH}_{2}, \mathrm{OCH}_{2} \mathrm{CH}_{2} \mathrm{~N}_{3}$, sugar $\mathrm{H}$ ), 2.14 (s, $3 \mathrm{H}$, $\mathrm{OAc}), 1.41(\mathrm{~s}, 3 \mathrm{H}, \mathrm{NHAc}) .{ }^{13} \mathrm{C} \mathrm{NMR}\left(\mathrm{CDCl}_{3}\right): \delta 171.0$ (2 C), 166.4, 166.0 ( $2 \times \mathrm{PhCO}, \mathrm{OAc}, \mathrm{NHAc}), 159.3$, $113.8\left(\mathrm{CH}_{3} \mathrm{OC}_{6} \mathrm{H}_{4} \mathrm{CH}_{2}\right), 138.7,138.6,138.4,138.3$, $133.4,130.4,129.9,129.7,128.6,128.4,128.3,128.2$, $128.1,128.0,127.9,127.5,127.3,126.4,126.3$ (Ar-C), 103.6, 102.5, 101.9, 100.6, 99.0 (C-1, C-1', C-1", C-1"', $\mathrm{PhCH}), 82.8,81.7,81.2,80.1,76.2,76.1,75.6,75.3$, 75.2, 75.0, 73.4, 73.3, 73.2, 73.1, 72.2, 71.7, 71.2, 70.0, 68.9, 68.5, 68.0, 66.3, 62.7 (sugar $\mathrm{C}, 5 \times \mathrm{PhCH}_{2}, \mathrm{PhCH}$, $\left.\mathrm{CH}_{3} \mathrm{O} \mathrm{C} \mathrm{H}_{6} \mathrm{H}_{4} \mathrm{CH}_{2}, \mathrm{OCH}_{2} \mathrm{CH}_{2} \mathrm{~N}_{3}\right), 55.3\left(\mathrm{CH}_{3} \mathrm{O}\right), 54.6(\mathrm{C}-$ $\left.2^{\prime \prime}\right), 51.0\left(\mathrm{OCH}_{2} \mathrm{CH}_{2} \mathrm{~N}_{3}\right), 23.6$ (NHAc), 20.9 (OAc). MALDI-TOFMS: $m / z$ 1708.9 [M + Na] ${ }^{+}, 1724.2[\mathrm{M}+$ $\mathrm{K}]^{+}$. Anal. Calcd for $\mathrm{C}_{94} \mathrm{H}_{100} \mathrm{~N}_{4} \mathrm{O}_{25}: \mathrm{C}, 66.98 ; \mathrm{H}, 5.94$; $\mathrm{N}, 3.32$. Found: C, 66.69; H, 6.21; N, 3.11.

\section{2-Azidoethyl 4-O-acetyl-2,6-di-O-benzoyl- $\beta$-D-} galactopyranosyl- $(1 \rightarrow 3)$-2-acetamido-4,6-O - benzylidene-2-deoxy- $\beta$-D-galactopyranosyl- $(1 \rightarrow 4)$ - 2,6-di-Obenzyl- $\beta$ - D-galactopyranosyl- $(1 \rightarrow 4)$ - 2,3,6-tri-O-benzyl- $\beta$-D-glucopyranoside (17). - To a solution of $\mathbf{1 6}$ $(700 \mathrm{mg})$ in $\mathrm{CH}_{3} \mathrm{CN}(27 \mathrm{~mL})$ and water $(3 \mathrm{~mL})$ was added ammonium cerium(IV) nitrate $(675 \mathrm{mg})$, and the mixture was stirred for $1.5 \mathrm{~h}$ at room temperature. TLC (1.3:1 petroleum ether-acetone) then showed the disappearance of $\mathbf{1 6}$ and the formation of $\mathbf{1 7}$. The mixture was diluted with $\mathrm{CH}_{2} \mathrm{Cl}_{2}(100 \mathrm{~mL})$ and washed with aq $\mathrm{NaHCO}_{3}(3 \times 50 \mathrm{~mL})$. The organic layer was dried, filtered, and concentrated. Column chromatography (1.3:1 petroleum ether-acetone) of the residue afforded
17, isolated as a colorless solid $(600 \mathrm{mg}, 92.4 \%) .[\alpha]_{\mathrm{D}}$ $+21.3\left(c 2.07, \mathrm{CHCl}_{3}\right) .{ }^{1} \mathrm{H}$ NMR $\left(\mathrm{CDCl}_{3}\right): \delta 8.04-6.81$ $(\mathrm{m}, 40 \mathrm{H}, \mathrm{Ar}-\mathrm{H}), 5.48-3.11(\mathrm{~m}, 43 \mathrm{H}$, sugar $\mathrm{H}, 5 \times$ $\mathrm{PhCH}_{2}, \mathrm{PhCH}, \mathrm{OCH}_{2} \mathrm{CH}_{2} \mathrm{~N}_{3}$ ), 2.09 (s, $3 \mathrm{H}, \mathrm{OAc}$ ), 1.30 (s, $3 \mathrm{H}, \mathrm{NHAc}) .{ }^{13} \mathrm{C}$ NMR $\left(\mathrm{CDCl}_{3}\right): \delta 171.4,171.0$, $165.9(2 \times \mathrm{PhCO}, \mathrm{OAc}, \mathrm{NHAc}), 103.5,102.2,101.9$, 100.4, 99.0 (C-1, C-1', C-1", C-1"', $\mathrm{PhCH}), 54.7$ (C-2"), $50.9\left(\mathrm{OCH}_{2} \mathrm{CH}_{2} \mathrm{~N}_{3}\right), 23.1$ (NHAc), 20.8 (OAc). Anal. Calcd for $\mathrm{C}_{86} \mathrm{H}_{92} \mathrm{~N}_{4} \mathrm{O}_{24}$ : C, 65.98; H, 5.88; N, 3.58. Found: C, 65.69; H, 6.17; N, 3.33.

2-Azidoethyl 4-O-acetyl-2,6-di-O-benzoyl-3-O-sulfo$\beta$-D-galactopyranosyl- $(1 \rightarrow 3)$-2-acetamido-4,6-O-benzylidene-2-deoxy- $\beta$-D-galactopyranosyl- $(\rightarrow 4)$ - 2,6 -diO-benzyl-3-O-sulfo- $\beta$-D-galactopyranosyl- $(1 \rightarrow 4)-2,3,6$ tri-O-benzyl- $\beta$-D-glucopyranoside (18). - To a solution of 17 (220 mg, $0.639 \mathrm{mmol})$ in dry pyridine was added sulfur trioxide pyridine complex (668 $\mathrm{mg}, 4.20 \mathrm{mmol}$ ), and the mixture was stirred at room temperature for 36 h. $\mathrm{MeOH}(1 \mathrm{~mL})$ was added, and stirring was continued for $10 \mathrm{~min}$. The mixture was concentrated, and the residue was purified by flash chromatography (10:1 $\left.\mathrm{CHCl}_{3}-\mathrm{MeOH}\right)$ to give $18(250 \mathrm{mg}, 94.3 \%) \cdot[\alpha]_{\mathrm{D}}+$ $43.4^{\circ}$ (c 1.29, $\left.\mathrm{MeOH}\right) .{ }^{1} \mathrm{H}$ NMR ( $\left.\mathrm{CD}_{3} \mathrm{OD}\right): \delta 8.12-7.13$ $\left(\mathrm{m}, \quad 40 \mathrm{H}, \quad\right.$ Ar-H), 5.85 (bs, $\left.1 \mathrm{H}, \mathrm{H}-4^{\prime \prime \prime}\right), \quad 5.46$ (t, $\left.1 \mathrm{H}, J_{2^{\prime \prime \prime} 3^{\prime \prime \prime}} 8.62 \mathrm{~Hz}, \mathrm{H}-2^{\prime \prime \prime}\right), 5.17(\mathrm{~s}, 1 \mathrm{H}, \mathrm{PhCH}), 5.16$ (d, $\left.1 \mathrm{H}, J_{1^{\prime \prime}, 2^{\prime \prime \prime}} 7.64 \mathrm{~Hz}, \mathrm{H}-1^{\prime \prime \prime}\right), 4.98$ (d, $\left.1 \mathrm{H}, \mathrm{H}-3^{\prime \prime \prime}\right)$, 4.88-4.18 (m, $18 \mathrm{H}), 3.96-3.80\left(\mathrm{~m}, 1 \mathrm{H}, \mathrm{OCH}_{2} \mathrm{CH}_{2} \mathrm{~N}_{3}\right)$, $3.80-3.34(\mathrm{~m}, 19 \mathrm{H}), 3.22\left(\mathrm{t}, 1 \mathrm{H}, J_{1,2} 8.10 \mathrm{~Hz}, \mathrm{H}-2\right)$. ${ }^{13} \mathrm{C}$ NMR $\left(\mathrm{CDCl}_{3}\right): \delta 174.9,172.3,167.8,167.7(2 \times$ PhCO, OAc, NHAc), 140.1, 140.0, 139.5, 139.4, 134.7, 1 ' $34.4,131.7,131.3,131.2,131.1,130.3,129.9,129.7$, $129.6,129.5,129.3,129.2,129.1,129.0,128.7,128.3$, 127.9, 127.7 (Ar-C), 104.7 (C-1', C-1"), 104.2 (C-1"'), 103.9 (C-1), $101.9(\mathrm{PhCH}), 83.9,82.9,81.9,79.6$, 78.0, 77.1, 76.7, 76.3, 76.1, 76.0, 75.3, 74.7, 74.2, 74.1, 72.5, 71.6, 71.0, 69.3, 69.2, 67.7, 67.5, 64.7 (sugar $\left.\mathrm{C}, \quad 5 \times \mathrm{PhCH}_{2}, \quad \mathrm{OCH}_{2} \mathrm{CH}_{2} \mathrm{~N}_{3}\right), \quad 52.2 \quad\left(\mathrm{C}-2^{\prime \prime}\right), \quad 49.9$ $\left(\mathrm{OCH}_{2} \mathrm{CH}_{2} \mathrm{~N}_{3}\right), 23.1$ (NHAc), 21.0 (OAc). MALDITOFMS: $m / z \quad 1742.8 \quad[\mathrm{M}-2 \mathrm{H}+\mathrm{Na}]^{-}, \quad 1758.9$ $[\mathrm{M}-2 \mathrm{H}+\mathrm{K}]^{-}$(negative-ion mode).

2-Aminoethyl 2-O-benzoyl-3-O-sulfo- $\beta$-D-galactopyranosyl- $(1 \rightarrow 3)$ - 2 -acetamido- 2 -deoxy- $\beta$ - D-galactopyranosyl- $(1 \rightarrow 4)$-3-O-sulfo- $\beta$-D-galactopyranosyl- $(1 \rightarrow 4)$ $\beta$-D-glucopyranoside (20). - A solution of $18(100 \mathrm{mg})$ in $10: 1 \mathrm{MeOH}-\mathrm{H}_{2} \mathrm{O}(15 \mathrm{~mL})$ and $\mathrm{HCl}(1 \mathrm{M}, 160 \mu \mathrm{L})$ was hydrogenolysed at $0.42 \mathrm{MPa}$ in the presence of palladium-on-charcoal $(10 \%, 100 \mathrm{mg})$ for $60 \mathrm{~h}$. The mixture was then filtered through Celite, and the solid was washed thoroughly with $\mathrm{MeOH}$ and water. The filtrate was then concentrated. Flash chromatography (5:4:0.6:1 $\left.\mathrm{CHCl}_{3}-\mathrm{MeOH}-\mathrm{H}_{2} \mathrm{O}-\mathrm{HOAc}\right)$ of the residue afforded 2-aminoethyl 4- $O$-acetyl-2,6-di- $O$-benzoyl-3- $O$ sulfo - $\beta$ - D - galactopyranosyl - $(1 \rightarrow 3)$ - 2 - acetamido - 2deoxy - $\beta$ - D - galactopyranosyl - $(1 \rightarrow 4)$ - 3 - $O$ - sulfo - $\beta$ - Dgalactopyranosyl-( $1 \rightarrow 4)-\beta$-D-glucopyranoside $(19, \quad 55$ $\mathrm{mg}, 88 \%)$ as a white solid. MALDI-TOFMS: $\mathrm{m} / \mathrm{z}$ $1184.2[\mathrm{M}-2 \mathrm{H}+\mathrm{Na}]^{-}$(negative-ion mode). 
To a solution of $19(50 \mathrm{mg})$ in dry $\mathrm{MeOH}$ was added $\mathrm{NaOMe}(10 \mathrm{mg})$. The mixture was stirred overnight at room temperature, then neutralized with HOAc until pH 7 was reached. The solution was then concentrated. Purification of the residue by passage through a Sephadex LH-20 column using water as eluent afforded, after lypophilization, 20 (44 mg, quant) as a white solid. $[\alpha]_{\mathrm{D}}+7.4^{\circ}\left(c 0.5\right.$, water). ${ }^{1} \mathrm{H}$ NMR $\left(\mathrm{D}_{2} \mathrm{O}\right): \delta 8.10-7.60$ (m, $5 \mathrm{H}, \mathrm{PhCO}), 5.32\left(\mathrm{t}, 1 \mathrm{H}, J_{2^{\prime \prime \prime} 3^{\prime \prime \prime}} 8.42 \mathrm{~Hz}, \mathrm{H}-2^{\prime \prime \prime}\right), 4.96$ $\left(\mathrm{d}, 1 \mathrm{H}, J_{1^{\prime \prime \prime}, 2^{\prime \prime \prime}} 7.61 \mathrm{~Hz}, \mathrm{H}-1^{\prime \prime \prime}\right), 4.71\left(\mathrm{dd}, 1 \mathrm{H}, J_{3^{\prime \prime \prime}, 4^{\prime \prime}} 3.30\right.$ $\left.\mathrm{Hz}, J_{2^{\prime \prime \prime}, 3^{\prime \prime \prime}} 6.47 \mathrm{~Hz}, \mathrm{H}-3^{\prime \prime \prime}\right), 4.57$ (d, $1 \mathrm{H}, J_{1^{\prime \prime}, 2^{\prime \prime}} 8.06 \mathrm{~Hz}$, H-1"), $4.53\left(\mathrm{~d}, 1 \mathrm{H}, J_{1^{\prime}, 2^{\prime}} 8.06 \mathrm{~Hz}, \mathrm{H}-1^{\prime}\right), 4.49(\mathrm{~d}, 1 \mathrm{H}$, $\left.J_{1,2} 7.69 \mathrm{~Hz}, \mathrm{H}-1\right), 4.34-4.31$ (m, $3 \mathrm{H}, \mathrm{H}-3, \mathrm{H}-4, \mathrm{H}-4^{\prime \prime \prime}$ ), $4.24\left(\mathrm{~d}, 1 \mathrm{H}, J_{3^{\prime \prime}, 4^{\prime \prime}} 2.20 \mathrm{~Hz}, \mathrm{H}-4^{\prime \prime}\right), 4.13-4.10(\mathrm{~m}, 1 \mathrm{H}$, $\mathrm{OCH}_{2} \mathrm{CH}_{2} \mathrm{NH}_{2}$ ), 3.96-3.71 (m, $\left.13 \mathrm{H}\right), 3.67-3.57$ (m, 9 $\mathrm{H}), 3.36\left(\mathrm{t}, 2 \mathrm{H}, \mathrm{H}-2, \mathrm{H}-2^{\prime}\right), 3.27(\mathrm{t}, 2 \mathrm{H}$, $\left.\mathrm{OCH}_{2} \mathrm{CH}_{2} \mathrm{NH}_{2}\right), 1.20$ (s, $\left.3 \mathrm{H}, \mathrm{NHAc}\right) .{ }^{13} \mathrm{C} \mathrm{NMR}$ $\left(\mathrm{D}_{2} \mathrm{O}\right): \delta 177.2$ (NHAc), 170.3 (PhCO), 137.1, 132.9, 131.7, 131.5 (Ar-C), 105.6 (C-1'), 105.4 (C-1), 105.2 $\left(\mathrm{C}-1^{\prime \prime \prime}\right), 104.8\left(\mathrm{C}-1^{\prime \prime}\right), 83.2\left(\mathrm{C}-3^{\prime \prime}\right), 82.1(\mathrm{C}-3), 81.2\left(\mathrm{C}-3^{\prime}\right)$, 80.7 (C-3"'), 77.0 (C-4), 75.5 (C-2), 73.3 (C-2"'), 72.1 $\left(\mathrm{C}-2^{\prime}\right), 70.6\left(\mathrm{C}-4^{\prime \prime}\right), 68.7\left(\mathrm{OCH}_{2} \mathrm{CH}_{2} \mathrm{NH}_{2}\right), 63.7$ (C-6, $\left.6^{\prime \prime \prime}\right), \quad 63.6 \quad\left(\mathrm{C}-6^{\prime}\right), \quad 63.4 \quad\left(\mathrm{C}-6^{\prime \prime}\right), \quad 53.6 \quad\left(\mathrm{C}-2^{\prime \prime}\right), \quad 42.3$ $\left(\mathrm{OCH}_{2} \mathrm{CH}_{2} \mathrm{NH}_{2}\right), 24.3$ (NHAc). MALDI-TOFMS: $m / z$ $1035.9[\mathrm{M}-2 \mathrm{H}+\mathrm{Na}]^{-}$(negative-ion mode).

2 -Aminoethyl 3-O-sulfo- $\beta$-D-galactopyranosyl- $(1 \rightarrow$ 3)-2-acetamido-2-deoxy- $\beta$-D-galactopyranosyl- $(1 \rightarrow 4)$ 3-O-sulfo- $\beta$-D-galactopyranosyl- $(1 \rightarrow 4)-\beta$-D-glucopyranoside (1). - A solution of $19(50 \mathrm{mg}, 0.0422 \mathrm{mmol})$ in $0.5 \mathrm{M} \mathrm{NaOMe}-\mathrm{MeOH}(10 \mathrm{~mL})$ was stirred at $0{ }^{\circ} \mathrm{C}$ for $6 \mathrm{~h}$. Work-up of the reaction mixture as described for compound $\mathbf{2 0}$ afforded $\mathbf{1}(35.2 \mathrm{mg}, 90 \%)$ as a white solid. $[\alpha]_{\mathrm{D}}+10.2^{\circ}\left(c 0.8\right.$, water). ${ }^{1} \mathrm{H}$ NMR $\left(\mathrm{D}_{2} \mathrm{O}\right): \delta$ $4.69\left(\mathrm{~d}, 1 \mathrm{H}, J_{1^{\prime \prime}, 2^{\prime \prime}} 8.52 \mathrm{~Hz}, \mathrm{H}-1^{\prime \prime}\right), 4.58\left(\mathrm{~d}, 1 \mathrm{H}, J_{1^{\prime \prime \prime}, 2^{\prime \prime}}\right.$ $\left.7.83 \mathrm{~Hz}, \mathrm{H}-1^{\prime \prime \prime}\right), 4.563$ (d, $\left.1 \mathrm{H}, J_{1^{\prime}, 2^{\prime}} 8.00 \mathrm{~Hz}, \mathrm{H}-1^{\prime}\right), 4.560$ $\left(\mathrm{d}, 1 \mathrm{H}, J_{1,2} 7.83 \mathrm{~Hz}, \mathrm{H}-1\right), 4.43\left(\mathrm{dd}, 1 \mathrm{H}, J_{3^{\prime}, 4^{\prime}} 2.92 \mathrm{~Hz}\right.$, H-4'), $4.40\left(\mathrm{dd}, 1 \mathrm{H}, J_{2^{\prime}, 3^{\prime}} 9.89 \mathrm{~Hz}, \mathrm{H}-3^{\prime}\right), 4.33$ (dd, $1 \mathrm{H}$, $\left.J_{3^{\prime \prime \prime}, 4^{\prime \prime}} 3.35 \mathrm{~Hz}, \mathrm{H}-3^{\prime \prime \prime}\right), 4.30$ (bs, $\left.1 \mathrm{H}, \mathrm{H}-4^{\prime \prime \prime}\right), 4.18$ (d, $1 \mathrm{H}$, $\left.J_{3^{\prime \prime}, 4^{\prime \prime}} \quad 3.10 \mathrm{~Hz}, \mathrm{H}-4^{\prime \prime}\right), \quad 4.15-4.12 \quad(\mathrm{~m}, 1 \mathrm{H}$, $\left.\mathrm{OCH}_{2} \mathrm{CH}_{2} \mathrm{NH}_{2}\right), 4.10-4.08(\mathrm{~m}, 1 \mathrm{H}), 4.07(\mathrm{dd}, 1 \mathrm{H}$, $\left.J_{2^{\prime \prime}, 3^{\prime \prime}} 10.88 \mathrm{~Hz}, \mathrm{H}-2^{\prime \prime}\right), \quad 4.01-3.98 \quad(\mathrm{~m}, 1 \mathrm{H}$, $\mathrm{OCH}_{2} \mathrm{CH}_{2} \mathrm{NH}_{2}$ ), 3.93 (dd, $1 \mathrm{H}, \mathrm{H}-3$ "), 3.87-3.62 (m, 15 $\mathrm{H}), 4.16-4.12\left(\mathrm{~m}, 1 \mathrm{H}, \mathrm{OCH}_{2} \mathrm{CH}_{2} \mathrm{NH}_{2}\right), 4.08(\mathrm{dd}, 1 \mathrm{H}$, $\mathrm{H}-2)$, 4.01-3.99 (m, $\left.1 \mathrm{H}, \mathrm{OCH}_{2} \mathrm{CH}_{2} \mathrm{NH}_{2}\right), 3.87-3.62$ (m, 15 H, H-2"', H-3, H-4, H-5, H-5', H-5", H-5"', H-6, H-6', H-6", H-6"'), 3.53 (dd, 1 H, H-2'), 3.38 (t, 1 H, $J_{2,3}$
$8.17 \mathrm{~Hz}, \mathrm{H}-2), 3.28$ (t, $2 \mathrm{H}, \mathrm{OCH}_{2} \mathrm{CH}_{2} \mathrm{NH}_{2}$ ), 2.10 (s, 3 $\mathrm{H}, \mathrm{NHAc}) .{ }^{13} \mathrm{C}$ NMR $\left(\mathrm{D}_{2} \mathrm{O}\right): \delta 177.8$ (NHAc), 107.3 (C-1"'), 105.4 (2 C, C-1, C-1"), 104.8 (C-1'), 83.0 (C-3"'), $82.7\left(\mathrm{C}-3^{\prime \prime}\right), 82.3\left(\mathrm{C}-3^{\prime}\right), 81.2(\mathrm{C}-3), 77.6\left(\mathrm{C}-2^{\prime \prime \prime}\right), 77.4$, 77.3, 77.0, 76.9, 76.6 (C-4', C-5, C-5', C-5", C-5"'), 75.5 (C-2), 75.3 (C-2'), 71.6 (C-4), 70.6 (C-4"), 69.6 (C-4"'), $68.7\left(\mathrm{OCH}_{2} \mathrm{CH}_{2} \mathrm{NH}_{2}\right), 63.8,63.7,63.5,62.7$ (C-6, C-6', C-6", C-6"'), 54.0 (C-2"), $42.3\left(\mathrm{OCH}_{2} \mathrm{CH}_{2} \mathrm{NH}_{2}\right), 25.3$ (NHAc). MALDI-TOFMS: $m / z 932.6[\mathrm{M}-2 \mathrm{H}+\mathrm{Na}]^{-}$ (negative-ion mode).

\section{Acknowledgements}

This project was supported by the National Natural Science Foundation of P. R. China (NSFC) and a grant from the Ministry of Science and Technology of P. R. China.

\section{References}

1. (a) Hakomori, S.; Zhang, Y. Chem. Biol. 1997, 4, 97-104; (b) Kim, Y. J.; Varki, A. Glycoconjugate J. 1997, 14, $569-576$.

2. Hakomori, S. Cancer Res. 1996, 56, 5309-5318.

3. Livingston, P. O. Immunol. Rev. 1995, 145, 147-166.

4. Tadano, K.; Ishizuka, I. J. Biol. Chem. 1982, 57, 1341313420 .

5. Hiraiwa, N.; Iida, N.; Ishizuka, I. Cancer Res. 1988, 48, 6769-6774

6. Hiraiwa, N.; Fukudas, Y.; Imura, H. Cancer Res. 1990 , 50, 2917-2928.

7. Chernyak, A.; Oscarson, S.; Turek, D. Carbohydr. Res. 2000, 329, 309-316.

8. Nashed, M. A.; Anderson, L. Carbohydr. Res. 1977, 56, 419-422.

9. Kameyama, A.; Ishida, H.; Kiso, M.; Hasegawa, A. Carbohydr. Res. 1990, 200, 269-285.

10. (a) Grundler, G.; Schmidt, R. R. Carbohydr. Res. 1985, 135, 203;

(b) Pougny, J. R.; Nasser, M. A. M.; Naulet, N.; Sinaÿ, P. Nouv. J. Chim. 1978, 2, 389.

11. Kanie, O.; Crawley, S. C.; Palcic, M. M.; Hindsgaul, O. Carbohydr. Res. 1993, 243, 139-164.

12. Sarbajna, S.; Roy, N. Carbohydr. Res. 1998, 306, 401407.

13. Spijker, N. M.; Keuning, C. A.; Hooglugt, M. Tetrahedron 1996, 52, 5945-5960.

14. Stahl, W.; Sprengard, U.; Kretschmar, G.; Kunz, H. Angew. Chem., Int. Ed. Engl. 1994, 33, 2096-2098. 